\title{
Size-mass-luminosity relations in AGN and the role of the accretion disc
}

\author{
S. Collin ${ }^{1}$ and J.-M. Huré1,2 \\ 1 DAEC/UMR 8631 du CNRS, Observatoire de Paris, Section de Meudon, 92195 Meudon, France \\ 2 Université Paris 7 Denis Diderot, Place Jussieu, 75251 Paris Cedex 05, France
}

Received 2 January 2001 / Accepted 21 March 2001

\begin{abstract}
We address the question of the relations between the black hole's mass, the accretion rate, the bolometric luminosity, the optical luminosity and the size of the Broad Line Region (BLR) in Active Galactic Nuclei, using recent observational data obtained from monitoring campaigns. We first show that a standard accretion disc cannot account for the observed optical luminosity, unless it radiates at super-Eddington rates. This implies the existence of another, dominant emission mechanism in the optical range, which could be due to the reprocessing of X-rays by a system of dense clouds, or a non standard disc (non stationary, ADAF and/or strong outflows). Narrow Line Seyfert 1 galaxies (NLS1s) are most extreme in this context: they have larger bolometric to Eddington luminosity ratios than Broad Line Seyfert 1 (BLS1s), and most likely a larger "non disc" component in the optical range. Second, from realistic simulations of self-gravitating $\alpha$-discs, we have systematically localized the gravitationally unstable disc and shown that, given uncertainties on both the model and observations, it coincides quite well with the size of the BLR. We therefore suggest that the gravitationally unstable disc is the source which releases BLR clouds in the medium. However, the influence of the ionization parameter is also required to explain the correlation found between the size of the BLR and the luminosity. In this picture, the size of the BLR in NLS1s (relative to the black hole size) is larger (and the emission line width smaller) than in BLS1s simply because their Eddington ratio is larger.
\end{abstract}

Key words. accretion, accretion disks - instabilities - galaxies: active - galaxies: nuclei

\section{Introduction}

There are several ubiquitous media in the central region of Active Galactic Nuclei (AGN): surrounding the supermassive black hole $(\mathrm{BH})$, there is an accretion disc $(\mathrm{AD})$, an X-ray source, a photoionized Broad Line Region (BLR) and possibly a molecular torus at the scale of a few parsecs from the center (Antonucci \& Miller 1985). Despite years of efforts, the physics of these components is far from being completely understood, nor is the possible interaction between them. For instance, the origin of the observed correlation between the size of the BLR and the luminosity of the AGN, which, under some hypothesis, translates into a relation between the mass of the central black hole and the disc accretion rate, is still mysterious.

In this paper, we try to understand the links between the accretion disc (at small and large radii), the optical, bolometric and Eddington luminosities, and finally the Broad Line Region (BLR). For this purpose, we use recent observational data which have led to the determination of the size of the BLR, optical luminosity, and black hole

Send offprint requests to: S. Collin, e-mail: suzy.collin@obspm.fr
(BH) mass in a few tens of objects spanning a large range of luminosity (Kaspi et al. 2000). In Sect. 2, we briefly recall the emission mechanisms in Active Galactic Nuclei (AGN), their link with the accretion disc, the main properties of the disc itself and those of the BLR. In Sect. 3 we discuss the relations between the observed optical luminosity, bolometric luminosity and BH mass. It is demonstrated that the standard disc emission cannot account for the optical luminosity, and that Narrow Line Seyfert 1 galaxies (NLS1s) are the most extreme cases on this point of view. In Sect. 4, we model the outer disc and show that the occurrence of gravitational instability roughly coincides with the size of the BLR. Our conclusions are presented in the last section.

\section{General considerations}

\subsection{Emission mechanisms in AGN: The inner disc}

Since its discovery in AGN spectra, the universal feature known as the "Big Blue Bump" (BBB), which represents the bulk of the bolometric luminosity, has been interpreted as thermal emission of an accretion disc (Shields 1978; Malkan \& Sargent 1982). If this disc is Keplerian and 


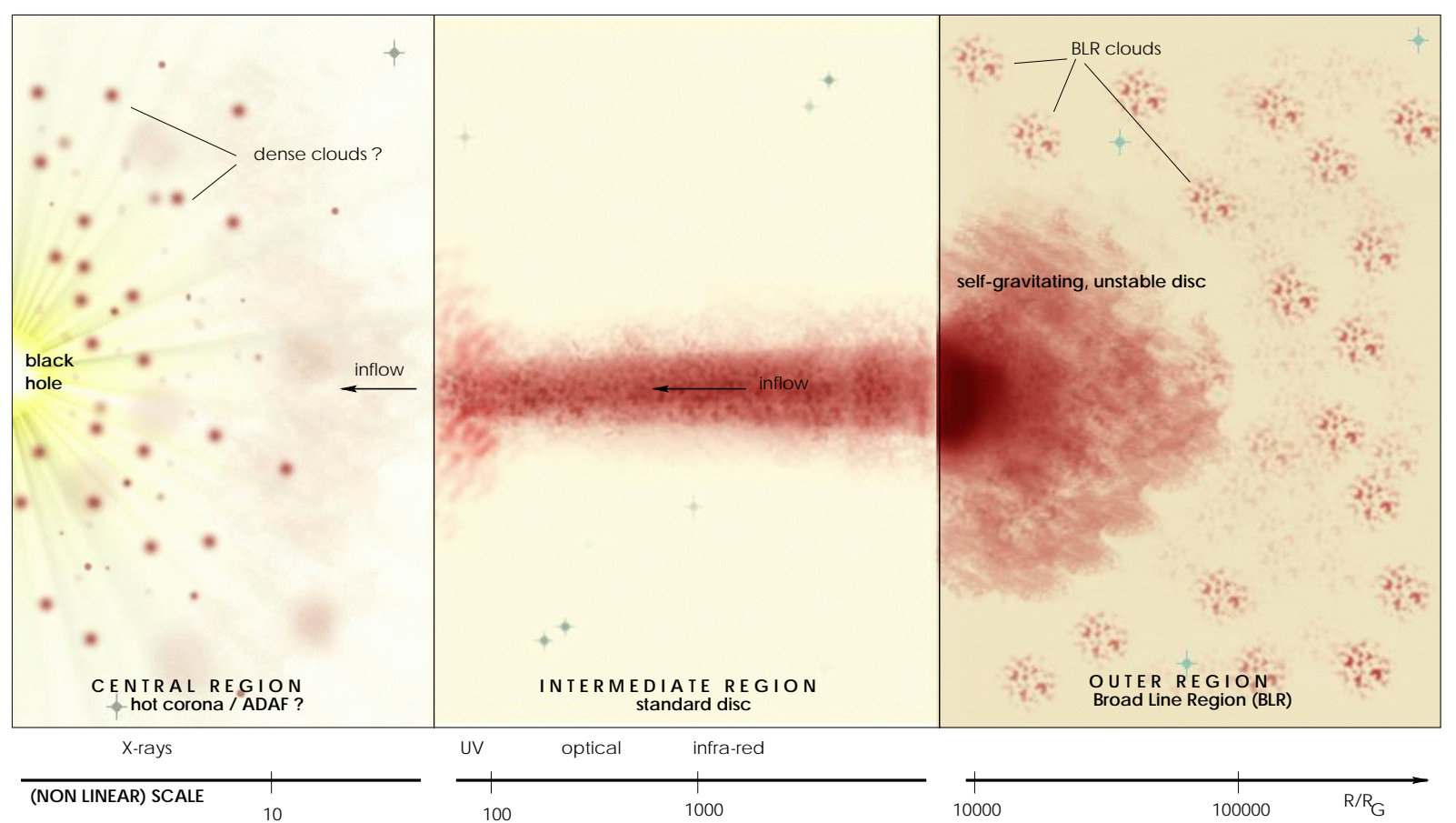

Fig. 1. Schematic view of the most central region of an AGN. The scaling depends slightly on the mass and on the accretion rate, and is appropriate for a $10^{8} M_{\odot}$ black hole $\left(R_{\mathrm{G}} \simeq 1.510^{13} \mathrm{~cm}\right)$ accreting at $\dot{m} \sim 0.1$ in Eddington units.

optically thick, as is generally assumed (the so-called "standard disc" model), then its emission reproduces roughly the BBB feature (see for a review Koratkar \& Blaes 1999; Collin 2000). Moreover, the idea that a non thermal power law continuum dominates the near infrared band and contributes, together with the disc, to the total emission in the optical range, has been abandoned after the publication of the generic spectrum of radio, quiet quasars by Sanders et al. (1989). These authors have actually shown that the IR band is most likely due to hot dust emission peaking at a few microns. Since hot dust sublimates at temperatures $\sim 1700 \mathrm{~K}$, dust cannot contribute to the optical emission. So, if one sustains the standard disc picture, one must admit that the optical continuum component is entirely emitted by the accretion disc.

According to the standard model, the disc regions emitting in the optical band are located at a few $100 R_{\mathrm{G}}$, where $R_{\mathrm{G}}=G M / c^{2}$ is the gravitational radius of the $\mathrm{BH}$ with mass $M$ (see Fig. 1, middle panel). Since the accretion luminosity varies as $R^{-1}$, the optical luminosity should be only a small fraction $(\sim 1 \%)$ of the bolometric luminosity. In contrast, the regions emitting the UV and EUV components are located close to the $\mathrm{BH}$, typically at distances of a few $10 R_{\mathrm{G}}$. Modeling the disc spectrum in the UV and EUV ranges is a difficult task as radiation is subject to several processes such as Comptonization. The emergent spectrum does depend strongly on the details of the disc vertical stratification, which is not well known. On the contrary, in the optical range, one can show that the local spectrum is roughly a blackbody at the effective temperature corresponding to the conversion of gravitational energy into radiation (Collin 2000).
The bolometric luminosity $L_{\text {bol }}$ is a robust prediction for a (steady state) standard disc: it is related only to the accretion rate $\dot{M}$ and to the radius of the last, dynamically stable orbit, and corresponds to an efficiency of massenergy conversion $\eta \approx 10 \%$ (the value adopted throughout this paper), depending on the spin of the black hole. Thus: $L_{\mathrm{bol}}=\eta \dot{M} c^{2}$,

and in principle, accretion stops if $L_{\mathrm{bol}}>L_{\mathrm{Edd}}$ where $L_{\mathrm{Edd}} \simeq 1.510^{45} M_{7} \mathrm{erg} / \mathrm{s}$ is the Eddington luminosity and $M_{7}$ is the mass in units of $10^{7} M_{\odot}$.

AGN spectra also display a hard X-ray spectrum which extends up to a few hundred $\mathrm{keV}$. This hard component which contributes a significant fraction of the bolometric luminosity (typically 30\%) cannot be produced by a bare standard disc. This is why one generally invokes the presence of a hot gas close to the BH (see Fig. 1, left panel), either in the form of a corona above the disc, or, instead of the inner disc itself, a quasi-spherical medium. In this case, the luminosities emitted respectively in the X-ray and optical-UV ranges relative to the accretion luminosity depend on the radius where this hot medium physically connects to the outer, standard disc.

The likely change in the morphology of the accretion flow in the inner region (thin disc $\rightarrow$ thick disc/spherical medium) is predicted by theory. The standard model probably does not work at small radii, where the disc is radiation pressure supported because of thermal instabilities. In comparison, the standard disc as a model for outer regions $\left(R \sim\right.$ a few $\left.10^{2} R_{\mathrm{G}}\right)$ seems more consistent: the disc is sustained by gas pressure, is relatively cold, and much more stable. It is true that a thermal instability, possibly recurrent (Siemiginovska et al. 1996), is 
expected where hydrogen recombines/ionizes but such an instability is predicted to keep the disc in a geometrically thin configuration.

\subsection{The status of the outer, self-gravitating disc}

At large radii (roughly $R \geq 10^{3} R_{\mathrm{G}}$; see below), the standard disc solution must be modified to account for the disc vertical self-gravity which may exceed the vertical component of the central gravity. The occurrence of vertical self-gravity is unescapable since it varies roughly as $R^{-3 / 5}$, i.e. less steeply than central gravity which varies as $R^{-3}$ (Shlosman \& Begelman 1989; Huré et al. 1994; Huré 1998). When the hydrostatic equilibrium is no longer controlled by the central object but by the disc, the surface density continuously increases whilst the disc gets thinner and thinner. But a self-gravitating disc is expected to be gravitationally unstable as soon as some criterion is fulfilled (Goldreich \& Lyndell-Bell 1965; see also Toomre 1964), usually this is $Q=\frac{\Omega^{2}}{\pi G \rho} \leq 1$, where $\Omega$ is the Keplerian angular velocity and $\rho$ is the local mass density.

The structure of the disc in the gravitationally unstable region is not known. It may become marginally stable again, if the viscosity self-adjusts in some fashion (LyndenBell \& Pringle 1974). Gravitational instabilities increase random (radial and vertical) velocities and could lead to the disc fragmentation into clouds (Pacynski 1968; Shore \& White 1982; Shlosman \& Begelman 1989). Following this scenario (see Fig. 1, right panel), it is often assumed that these clouds are moving with a high velocity, and that their chaotic motion and mutual collisions provide the support against vertical gravity and keep accretion at the required rate (Begelman \& Krolik 1986; Kumar 1999). The clumpy disc could become geometrically thick again, and even, at very large distance $(\sim 1 \mathrm{pc})$, it could connect to the dusty molecular torus invoked in the Unified Scheme of Seyfert nuclei (Antonnucci \& Miller 1985). It is also possible that fragments in the unstable disc collapse to form protostars. Because these protostars can trap a large amount of gas from the disc, they should rapidly evolve into massive stars, and then give rise to outflowing gas through winds and supernovae explosions (Collin \& Zahn 1999). In both scenarios one would thus expect the presence of a dense medium above the accretion disc, with an azimuthal velocity close to the Keplerian one, which could be identified with the BLR.

\subsection{Size and velocities within the $B L R$}

A major tool for studying the structure of the BLR is reverberation mapping, through the study of correlated variations of the lines and continuum fluxes (see the review by Peterson 1993). It has been used to determine the size of the BLR in several tens of AGN, and to correlate the mass of the central black hole with the luminosity of the AGN, under the assumption that the BLR is gravitationally bound to the black hole.
Though powerful for size determination, reverberation mapping does not provide the velocity field of the BLR, and can generally not distinguish between radial and rotational motions. In some objects however, there are indications that rotational motions of the medium emitting the $\mathrm{H} \beta$ line dominates over radial motions. On the other hand, the medium emitting high ionization lines (or HILs) is sometimes observed as an outflowing medium. This is thought to be the case in NGC 4051 (Peterson et al. 2000). However, it is likely that the velocity $V_{\mathrm{BLR}}$ does not differ strongly from a gravitationally bound, Keplerian or virial motion (Peterson \& Wandel 1999). Then, the radius of the BLR is approximately given by

$\frac{R_{\mathrm{BLR}}}{R_{\mathrm{G}}}=\frac{G M}{V_{\mathrm{BLR}}^{2}} \times \frac{c^{2}}{G M} \sim \frac{900}{V_{9}^{2}}$

where $V_{\mathrm{BLR}}=V_{9} 10^{9} \mathrm{~cm} / \mathrm{s}$.

Because there is no consensus about the dynamics of the BLR, there has been little progress in understanding the physics of this region. The most commonly accepted picture, deduced from photoionization models, consists of an assembly of photoionized clouds that cover at least $10 \%$ of the source emitting the incident, primary continuum. The number of BLR clouds is probably very large because the volumic filling factor of the emitting medium is extremely small. This large population ensures a large total emitting area as well as a high density and a small individual size. The smoothness of the line profiles also requires a large number of clouds. If these clouds are not confined by any mechanism, they expand in a very short time scale (less than one year). If the BLR is stationary, new clouds must be permanently generated. Alternatively, if the clouds are formed uniquely then some form of confinement mechanism must operate. The thermal confinement by a hot medium, as proposed in the "two phase model" by Krolik et al. (1981), implies a Thomson thick hot medium, which is difficult to reconcile with the very short variation time scale of the X-ray flux.

A natural idea which does not address the confinement problem is that the BLR is made of the atmosphere of giant or "bloated stars", or by winds from giant stars (Edwards 1980; Penston 1988; Scoville \& Norman 1988; Alexander \& Netzer 1994). This model however requires a very large number of stars to account both for the line luminosity and for the smoothness of the line profiles. Another possibility that does not appeal to any special confinement mechanism either is that the lines are emitted by the accretion disc. Dumont \& Collin-Souffrin (1990) have proposed that low ionization lines (or LILs), like Balmer lines, which require a high density emitting medium (Collin-Souffrin et al. 1986) are formed at the surface of the accretion disc. On the other hand, Murray \& Chiang $(1995,1997)$ proposed that the broad emission lines are emitted by a wind released at the top of the disc where motions are still mainly rotational. In this model, the broad absorption lines (BALs) are produced by the high-velocity component of the wind. In both cases, the BLR is dominated by rotation and its presence (or at least, 
that of the region emitting the LILs) is linked to the accretion disc.

\section{Relation between the optical luminosity and the bolometric luminosity}

\subsection{Global investigation}

Kaspi et al. (2000) combined their own reverberation mapping study with other published data to determine the size of the BLR, the BH mass and the optical luminosity. As is usually done (Peterson 1993, 2000), they derived the size of the BLR from the measurements of the time lags between the light curves relative to the $\mathrm{H} \alpha$ and $\mathrm{H} \beta$ fluxes and the light curve of the underlying continuum which is assumed to follow the ionizing continuum. From the size of the BLR, they computed the BH mass from the FWHM of $\mathrm{H} \beta$ and $\mathrm{H} \alpha$, assuming that these lines are emitted by a gravitationally bound medium. There is some uncertainty in the mass determination (by a factor of a few) since there are two different, non-equivalent methods to determine the $F W H M$ of lines. The $F W H M$ can be averaged on all spectra, giving a "mean" BH mass: this is the method preferred by Kaspi et al. (2000). In the method proposed by Peterson et al (1998), the FWHM is measured on the rms spectrum, giving a "rms" mass.

To estimate the bolometric luminosity from the monochromatic luminosity, $L_{\mathrm{bol}} \propto \nu L_{\nu}^{\mathrm{obs}}(5100 \AA)$ is generally assumed. For the AGN generic spectrum (Laor et al. 1997), one deduces:

$L_{\text {bol }} \sim 9 \times \nu L_{\nu}^{\text {obs }}(5100 \AA)$.

For each object in Kaspi et al. sample, we have compared this optical-to-bolometric conversion with two non equivalent but both self-consistent methods of conversion based on the disc model:

1. by computing the theoretical, monochromatic luminosity $\nu L_{\nu}^{\text {disc }}(5100 \AA)$ from the spectrum of an optically thick, steady state disc given the pair $(M, \dot{M})$, assuming Eq. (3), and Eq. (1) to estimate $\dot{M}$. Figure 2 displays the ratio $L_{\nu}^{\text {disc }}(5100 \AA) / L_{\nu}^{\text {obs }}(5100 \AA)$ obtained with this method. It shows that, for all objects in the sample, the emission due to the disc can represent only a minor, and in most cases, a negligible fraction of the observed luminosity in the optical band;

2. by finding the "right" accretion rate $\dot{M}$ (and then $L_{\text {bol }}$ via Eq. (1)) such that the observed monochromatic luminosity is only due to a standard disc (that is $\left.L_{\nu}^{\text {disc }}(5100 \AA)=L_{\nu}^{\text {obs }}(5100 \AA)\right)$. This method leads to extremely small ratios $\nu L_{\nu}^{\text {obs }}(5100 \AA) / L_{\text {bol }}$, as shown by Fig. 3. It implies a very large bolometric luminosity, and therefore a very large Eddington ratio $\dot{m}=L_{\text {bol }} / L_{\text {Edd }}$. To illustrate this last point, the corresponding Eddington ratio is plotted versus the central mass in Fig. 4. It shows that almost all the objects would be radiating at highly super-Eddington rates, proving that this second self-consistent opticalto-bolometric conversion is wrong.

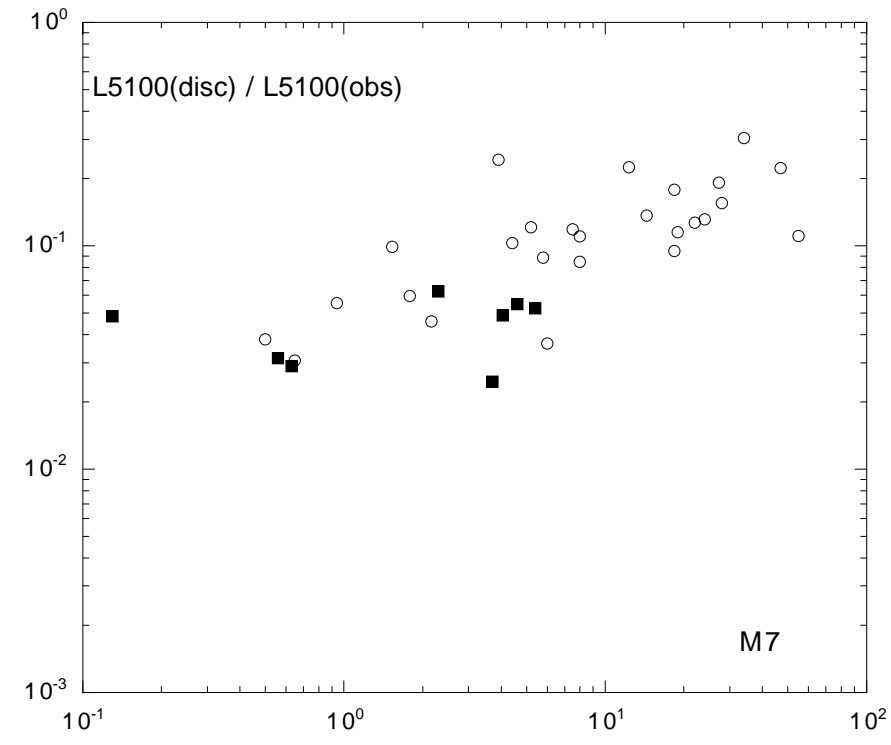

Fig. 2. $L_{\nu}^{\text {disc }}(5100 \AA) / L_{\nu}^{\text {obs }}(5100 \AA)$ ratio versus the mass (in units of $10^{7} M_{\odot}$ ) for the Kaspi et al. (2000) sample: $L_{\nu}^{\text {disc }}(5100 \AA)$ have been computed using Eqs. (1) and (3). We have distinguished NLS1 galaxies (squares) and other nuclei (circles).

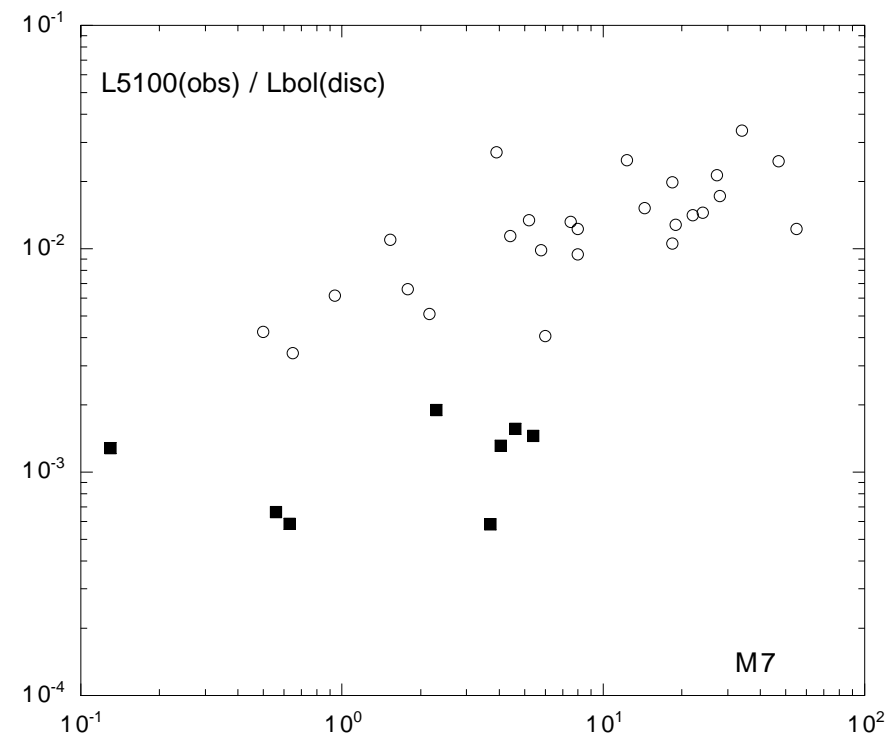

Fig. 3. $L(5100 \AA)_{\text {obs }} / L_{\text {bol }}$ ratio versus the mass (in units of $\left.10^{7} M_{\odot}\right)$ for the Kaspi et al. (2000) sample: $L_{\text {bol }}$ has been computed assuming that the optical luminosity $L(5100 \AA)_{\text {obs }}$ is entirely due to the disc. We have distinguished NLS1 galaxies (squares) and other nuclei (circles).

In other words, a relation of the form Eq. (3) should be roughly valid, meaning that the optical luminosity is not radiated by a standard disc.

Note that we assume a face-on disc, thus overestimat-

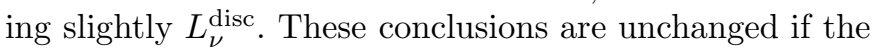
inner disc is removed for some reason, that is, if the inner radius is larger (in the limit of a few $10 R_{\mathrm{G}}$ ) than a few $R_{\mathrm{G}}$ as considered here, since the optical emission is almost entirely produced in outer regions located much further away from the center. Clearly the situation becomes even 


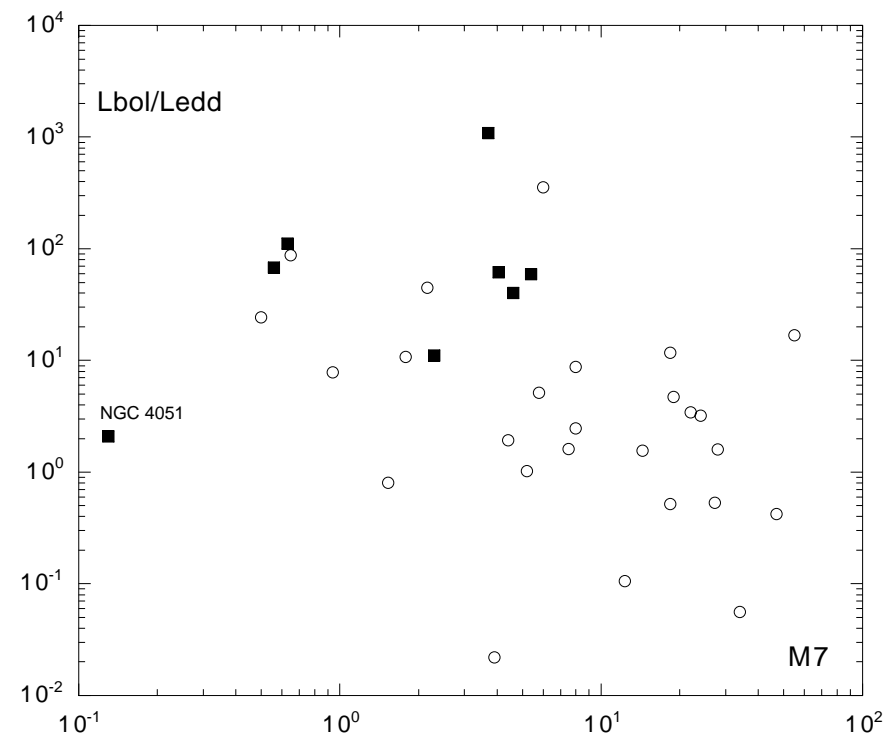

Fig. 4. $L_{\text {bol }} / L_{\text {Edd }}$ ratio versus the mass (in units of $10^{7} M_{\odot}$ ), for the Kaspi et al. (2000) sample, assuming that the optical monochromatic luminosity $L^{\text {obs }}(5100 \AA)$ is entirely due to the disc. We have distinguished NLS1 galaxies (squares) and other nuclei (circles). Note the point corresponding to the lowest mass is NGC 4051.

more extreme if the region emitting the optical band is not present in the disc, that is, if the disc is truncated for some reason, for instance due to self-gravity.

There are at least four possible explanations for this result:

1. the optical luminosity is not due to the disc, as quoted. This is plausible since the standard disc spectrum is well known to be flatter than the spectrum of Seyfert 1 nuclei in the optical range (Koratkar \& Blaes 1999; Hubeny et al. 2000; Collin 2000). This is also compatible with a recent study of the NLS1 1 RE J1034+396 by Puchnarewicz et al. (2000) who conclude that an additional underlying power law connecting the IR to the X-rays is really necessary to fit the optical/UV continuum. They suggest that this extra component might be a non thermal BLac-type component. This is indeed possible if for instance there is a strong magnetic field anchored into the disc. But it could also be due to a thermal process, like the emission of dense clouds surrounding the X-ray source and reprocessing a large fraction of the X-ray luminosity, as proposed by Celotti et al. (1992), and by Collin-Souffrin et al. (1996). This cloud system should be distributed quasispherically in order to reprocess a large fraction of the $\mathrm{X}$-ray luminosity (in the case of a disc, the reprocessed fraction decreases as $R^{-1}$, like the accretion luminosity, and is necessarily small in the optical range);

2. the standard disc model is not valid. Although the accretion luminosity does not depend on viscosity, the fact that the disc is effectively optically thick and radiates like a black body is not independent of the viscosity prescription. Note, as a proof, that the optical thickness of a standard disc is a function of the $\alpha$-viscosity parameter. Either a completely different mechanism of angular momentum evacuation in the disc is at work (e.g. density waves), or the formalism for turbulent viscosity must be reconsidered, or both;

3. the accretion rate is not uniform. This possibility includes the presence of a non steady disc and the possible existence of some steady inflow involving an accretion rate decreasing with decreasing radius. Concerning the first point, there are a few well known sources of instability that can perturb the equilibrium of a disc but this would mean that all objects are observed in a "high optical state". This is statistically somewhat doubtful. Regarding the second point, this would mean that a huge amount of gas is accreted at large distances and then converted into an outflow at smaller radii. This is also unrealistic because it would imply massive super-Eddington outflows, which are not observed;

4. accretion is advection dominated in the inner regions. Such a solution is still not demonstrated on theoretical grounds. Stable Advection Dominated Accretion Flows (ADAFs) (Narayan \& Yi 1995, and many other works) take place for relatively low accretion rates, well below the Eddington limit. At the other extreme, there are advective solutions for Eddington rates ("slim discs" for instance, cf. Abramowicz et al. 1988) or for superEddington rates (thick discs), but it is difficult to conceive a durable, super-Eddington accretion rate, as it would imply a dramatic growth of the hole over cosmic time scales. In conclusion, even if the disc becomes geometrically thick in the inner regions, advection is not likely to be important in the Seyfert galaxies and moderately luminous quasars considered here, and the gas should flow all the way down to the last stable orbit and radiate accordingly.

\subsection{The case of Narrow Line Seyfert 1}

Figure 5 displays the size of the BLR, $R_{\mathrm{BLR}}$, versus $\nu L_{\nu}^{\text {disc }}(5100 \AA)$ for the Kaspi et al. (2000) sample. In this figure and in Figs. 2-4, NLS1 have been isolated ${ }^{1}$. We notice that they do not occupy any privileged region in Fig. 5, contrary to Figs. 2-4. In Fig. 4 they are concentrated in the top-left region corresponding to small masses and high bolometric luminosities. Further, NLS1s have the smallest $L_{\nu}^{\text {disc }}(5100 \AA) / L_{\nu}^{\text {obs }}(5100 \AA)$ ratios compared to other objects in the same range of $\mathrm{BH}$ mass, as shown by Fig. 2. How can we explain these properties?

We know that NLS1 galaxies have a strong soft X-ray excess compared to Broad Line Seyfert 1 galaxies (BLS1s) (Boller et al. 1996 for a review; see also the proceedings edited by Boller et al. 2000). This would

\footnotetext{
${ }^{1}$ We define NLS1 as Seyfert nuclei with $V_{\mathrm{BLR}}=$ $\left(G M / R_{\mathrm{BLR}}\right)^{1 / 2}<2000 \mathrm{~km} \mathrm{~s}^{-1}$, instead of using $F W H M \mathrm{~s}$, to avoid having to take into account a correction for $\mathrm{H} \alpha$ and $\mathrm{H} \beta$.
} 


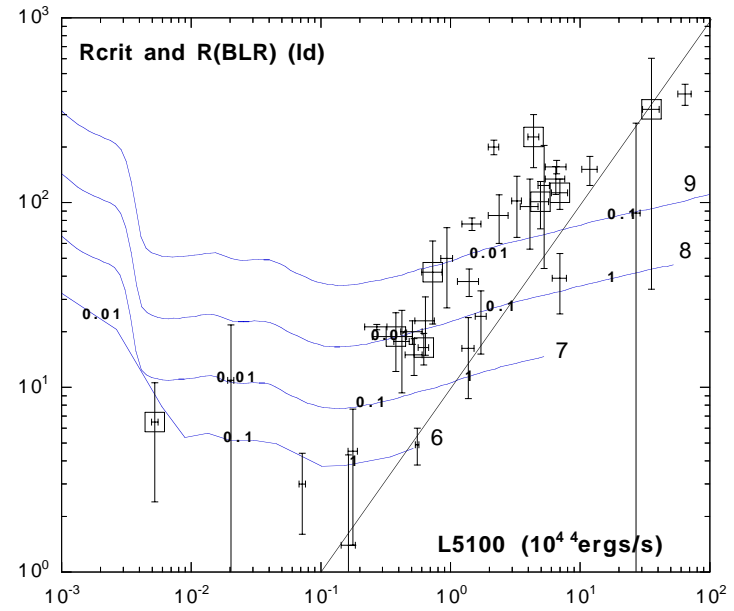

Fig. 5. $R_{\mathrm{BLR}}$ in lt-days versus the monochromatic luminosity at $5100 \AA$ in units of $10^{44} \mathrm{erg} \mathrm{s}^{-1}$, for the Kaspi et al. (2000) sample. The big open squares show NLS1s. The curves give $R_{\text {crit }}$ from the $2 \mathrm{D}$ simulations. The accretion rates of the theoretical curves have been converted into $L 5100$ assuming that the bolometric luminosity is equal to $9 \times L 5100$. The curves are labeled with $\log \left(M / M_{\odot}\right)$, and the Eddington ratio $\dot{m}$ is indicated on the curves.

suggest that the BBB is shifted towards the EUV, and that the ratio $L^{\text {disc }}(5100 \AA) / L^{\text {disc }}($ EUV $)$ is smaller in NLS1s than in BLS1s. Meanwhile, this would mean that the ratio $L_{\mathrm{bol}} / L^{\mathrm{obs}}(5100 \AA)$ is larger in NLS1s than in BLS1s. However this is impossible, as it would imply super-Eddington rates. Indeed Fig. 8, which displays $R_{\mathrm{BLR}} / R_{\mathrm{G}}$ as a function of the Eddington ratio, for $L_{\mathrm{bol}} / L^{\text {obs }}(5100 \AA)=9$, shows that with the conservative assumption of Eq. (3), NLS1s are already radiating at Eddington rates. NLS1s occupy a privileged position on this figure, not only because of their small $F W H M$ (corresponding by definition to large $R_{\mathrm{BLR}} / R_{\mathrm{G}}$ values), but also because they display large Eddington ratios. Actually this was strongly suspected on the basis of their spectral and variability properties in the X-ray range, similarly to galactic BH candidates (Pounds et al. 1995).

We conclude that NLS1s have not only larger Eddington ratios, but also probably a larger "non disc" fraction of optical emission, compared to BLS1s.

\section{What determines the size of the BLR?}

\subsection{Model for the outer disc}

Based mainly on distance arguments, we have suggested before that the BLR is possibly related to the suppression of the disc by gravitational instabilities. We have investigated this idea more quantitatively by a series of bi-dimensional simulations of a steady state, Keplerian accretion $\alpha$-disc (Huré 2000). More precisely, the model includes convection in the framework of the Mixing Length Theory, turbulent pressure that substantially thickens the disc and self-gravity within the infinite disc approximation. Opacities and equation of state are realistic and
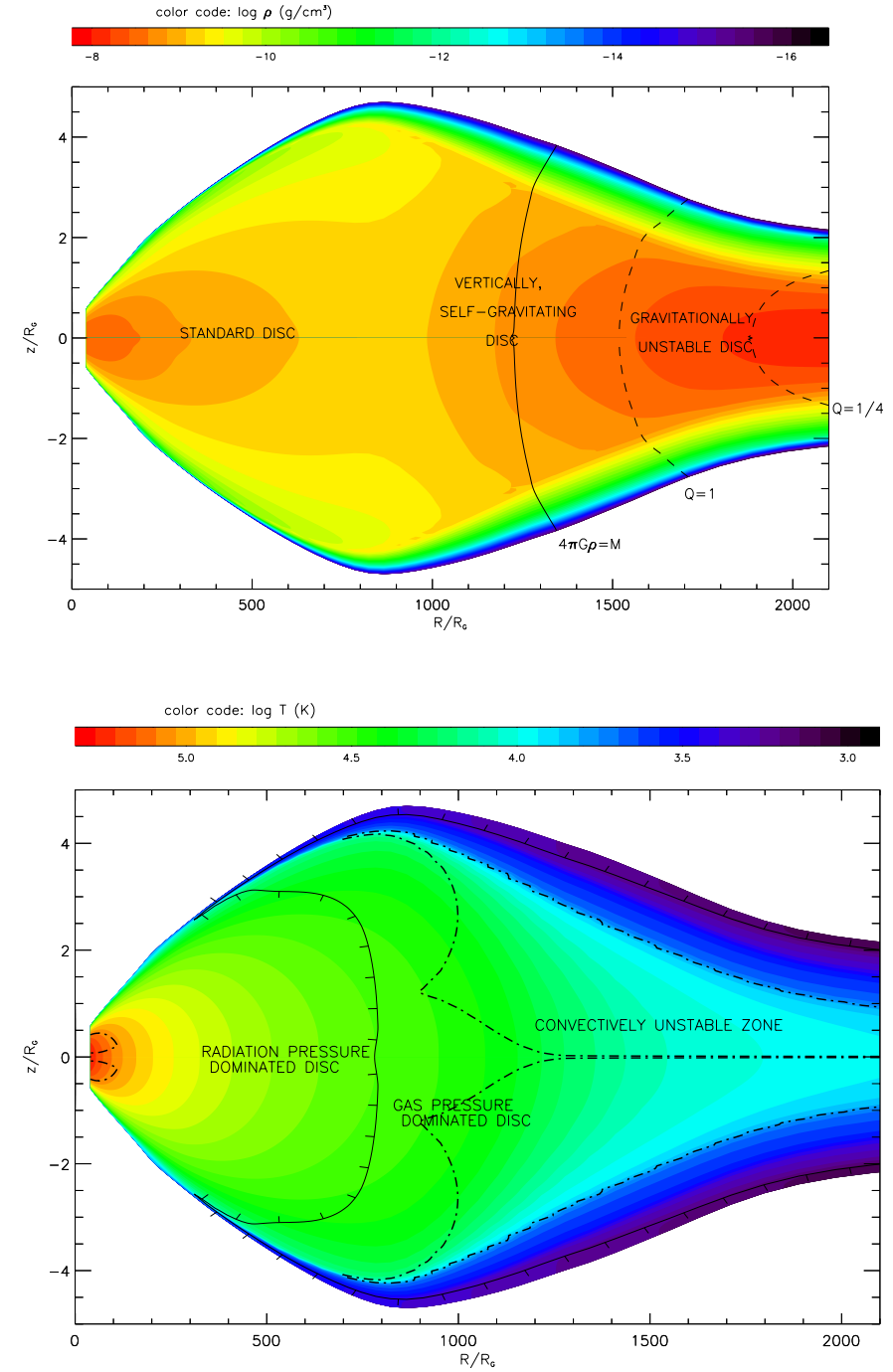

Fig. 6. Density map (top) and temperature map (bottom) for a steady state Keplerian $\alpha$-disc, surrounding a $10^{8} M_{\odot}$ black hole, versus $R$ and $z$ both normalized to $R_{\mathrm{G}}$. The accretion rate is $\dot{M}=10^{-1} M_{\odot} /$ yr (i.e. $\dot{m} \sim 0.03$ ), and $\alpha=0.1$.

corresponds to a gas at LTE with cosmic abundances. External irradiation is not taken into account, for several reasons. First, the discs considered here are optically very thick and moreover the irradiation flux is likely to decrease as the gravitational flux, having thus a relatively small influence on the disc structure (Collin \& Huré 1999). Second, as we shall see below, outer regions are in the shadow of the central regions due to self-gravity which pinches the disc vertically. Figure 6 shows an example of internal structure of a disc computed for $M=10^{8} M_{\odot}$, $\dot{M}=10^{-1} M_{\odot} /$ yr and $\alpha=0.1$. We see that the disc becomes self-gravitating (in the sense $4 \pi G \rho R^{3} \geq M$ ) from $\sim 1200 R_{\mathrm{G}}$ which results in a significant decrease of the disc thickness and rise of the density. Note however that the disc flaring vanishes at much lower radius $\sim 900 R_{\mathrm{G}}$. 


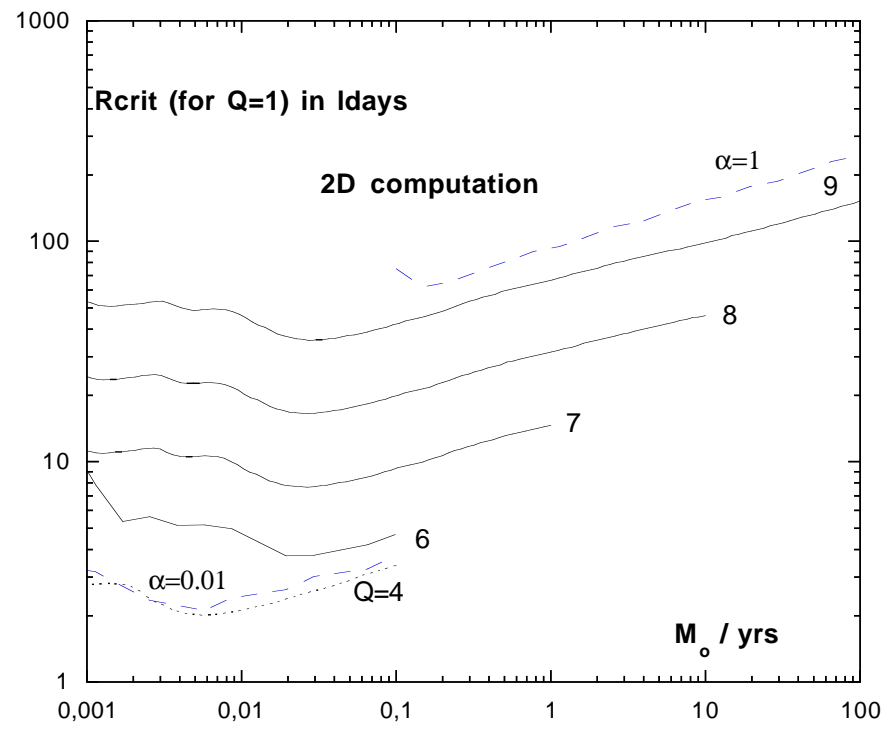

Fig. 7. $R_{\text {crit }}$ for $Q=1$ as a function of the accretion rate expressed in $M_{\odot} /$ yr. The curves are labeled with $\log \left(M / M_{\odot}\right)$. $\alpha=0.1$ throughout. A few other cases are also displayed (for $\alpha=0.01$ and $\alpha=1$, and for $Q=4$ ).

\subsection{Location of the gravitationally unstable region}

In order to determine the radius $R_{\text {crit }}$ where the disc becomes gravitationally unstable, we have computed a series of disc models by varying $M$ and $\dot{M}$. There are many possible sources of uncertainties on the value of this quantity, including a lack of information regarding some physical processes like turbulent viscosity through the $\alpha$-prescription and its $z$-dependency, convective transport, self-gravity, environment effects. In addition, the threshold value of the $Q$-parameter which defines the instability criterion is uncertain in this context (e.g., it is not clear whether this criterion applies to the midplane quantities). We have attempted to estimate an absolute error on $R_{\text {crit }}$ by considering $0.01 \leq \alpha \leq 1$ and $\frac{1}{4} \leq Q \leq 4$ (Toomre 1964; Goldreich \& Lynden-Bell 1965). Figure 6 shows the location of the unstable disc region $\left(R_{\text {crit }} \sim 1500-2000 R_{\mathrm{G}}\right)$. Our results are displayed in Fig. 7 which gives $R_{\text {crit }}$ versus the accretion rate for four mass decades, obtained for $\alpha=0.1$ and $Q=1$ (mean values). We see that $R_{\text {crit }}$ is mainly determined by the central mass and is weakly sensitive to the accretion rate $\dot{M}$. The $\alpha$-parameter and threshold value for $Q$ have rather minor effects for moderate and high Eddington luminosities. The magnitude of the error on $R_{\text {crit }}$ is given by the gap between curves corresponding to the same central mass but to different values of $\alpha$ and threshold values for $Q$. It is comforting to see that the results are quite reliable, as the uncertainties do not produce an "error" larger than a factor of say, $\sim 3$ on $R_{\text {crit }}$.

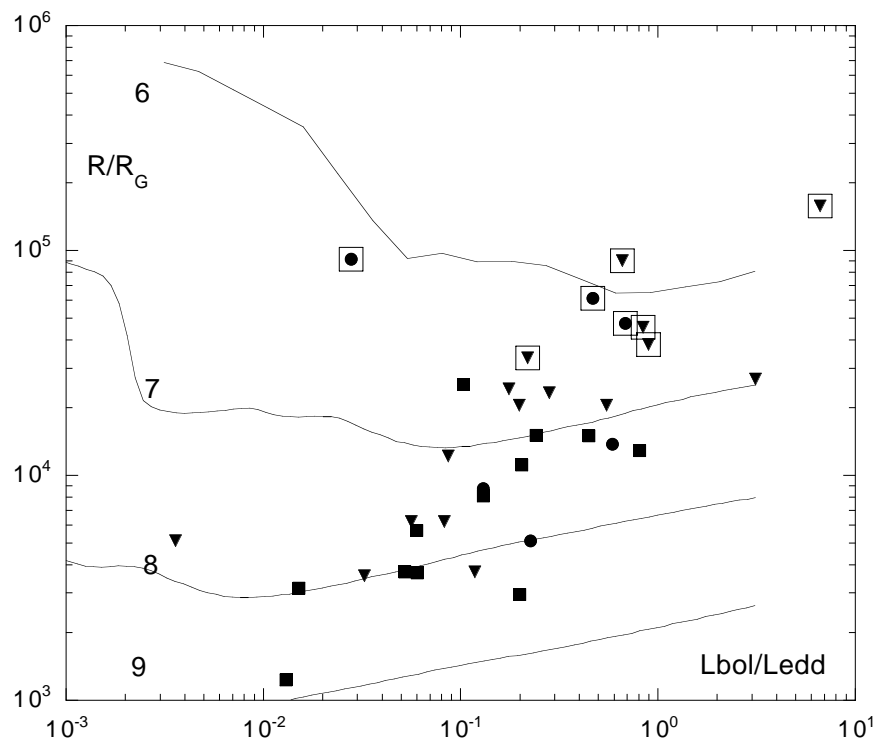

Fig. 8. The points give $R_{\mathrm{BLR}} / R_{\mathrm{G}}$ versus $L_{\mathrm{bol}} / L_{\mathrm{Edd}}$, binned according to the BH mass, and assuming $L_{\text {bol }}=9 \times L(5100 \AA)$, for the Kaspi et al. (2000) sample. Circles: $M \leq 10^{7} M_{\odot}$; triangles: $10^{7} \leq M \leq 10^{8} M_{\odot}$; squares: $M \geq 10^{8} M_{\odot}$. The big open squares are NLS1s. The curves give $R_{\text {crit }} / R_{\mathrm{G}}$ from the $2 \mathrm{D}$ simulations. They are labeled with $\log \left(M / M_{\odot}\right)$.

Figure 8 displays $R_{\text {crit }} / R_{\mathrm{G}}$ versus the Eddington ratio for the same models. A crude fit in the domain $0.01 \leq$ $\dot{m} \leq 1$ gives

$\frac{R_{\text {crit }}}{R_{\mathrm{G}}} \approx 210^{4} M_{7}^{-0.46}$.

\subsection{Comparing the BLR and the disc sizes}

We have plotted in Fig. 5 the $R_{\text {crit }}$ in light-days versus $L(5100 \AA)$, using Eq. (3) to convert the disc accretion rate into optical luminosity (otherwise, accretion rates are unrealistically high), together with the data of the Kaspi et al. sample. Figure 8 displays $R_{\mathrm{BLR}} / R_{\mathrm{G}}$, for the Kaspi et al. sample, still assuming Eq. (3). We see clearly from these two figures that, in absolute, the BLR size is correlated with the occurrence of the disc gravitational instability. So, if we identify the edge of the gravitationally unstable disc with the inner edge of BLR, that is $R_{\text {crit }} \leq R_{\mathrm{BLR}}$, we conclude that active nuclei hosting low mass black holes are predicted to have broad lines narrower than others.

Figure 8 shows that, though the observed masses do not match exactly the theoretical expectations, there is a clear trend that objects with small masses $\left(10^{6}<M<\right.$ $10^{7} M_{\odot}$ ) have a large $R_{\mathrm{BLR}} / R_{\text {crit }}$ ratio and that objects with large masses $\left(M>10^{8} M_{\odot}\right)$ have a small $R_{\mathrm{BLR}} / R_{\text {crit }}$ ratio, in agreement with our theoretical predictions. So, at least to this "zero order approximation", the gravitationally unstable region of the disc has kinematic and size properties compatible with the BLR and could therefore be the ultimate source of the BLR clouds. It is however difficult to draw more quantitative conclusions from this investigation. 


\subsection{Influence of the ionization parameter of the $B L R$}

One can notice that $R_{\text {crit }}$ depends mainly on the $\mathrm{BH}$ mass and little on the luminosity, while a correlation appears in Fig. 5 between $R_{\mathrm{BLR}}$ and the $L^{\mathrm{obs}}(5100 \AA)$. Kaspi et al. (2000) represent this correlation as $R_{\mathrm{BLR}} \propto$ $L(5100 \AA)^{0.70}$. A smaller power law index (closer to 0.5$)$ was found in previous works (e.g. Wandel et al. 1999). It is not clear how to translate such a relation into a relation between $R_{\mathrm{BLR}}$ and $L_{\mathrm{bol}}$, because of an important uncertainty arising from the optical-to-bolometric conversion. If the optical to bolometric luminosity ratio is constant, it results in $R_{\mathrm{BLR}} \propto L_{\mathrm{bol}}^{0.5-0.7}$.

In a recent paper, Nicastro (2000) proposed that the BLR clouds are released by the accretion disc in the region where a vertically outflowing corona exists, according to a model proposed by Witt et al. (1997). This is indeed an interesting model, since in this case the main parameter governing the size of the BLR would be $\dot{m}$ and not $M$. However one would expect that the remote regions giving rise to the BLR, which are strongly pressurized by the corona, are also gravitationally unstable. Moreover there is also another important parameter governing the size of the BLR, that is its ionization parameter

$\xi=\frac{L_{\text {ion }}}{n R_{\mathrm{BLR}}^{2}}$

where $L_{\text {ion }}$ is the ionizing luminosity $\left(\sim L_{\mathrm{bol}}\right)$ and $n$ is the number density in the clouds. Actually, any model must reproduce not only the kinematics and dimension of the BLR but also the ionization parameter (linked to the ionization state) consistent with the observed line ratios which are almost independent of the luminosity over more than four decades (except for the Baldwin effect which might indicate a decrease of the ionization parameter with increasing luminosity, but only for high ionization lines). Typically, $\xi \approx 1$ and detailed studies of the line ratios show that $n \approx 10^{10} \mathrm{~cm}^{-3}$. If the density is about a constant, the constancy of $\xi$ translates into a relation $R_{\mathrm{BLR}} \propto L_{\mathrm{bol}}^{0.5}$, in close agreement with the observations. Another way to express this relation is that the flux irradiating the clouds is constant. This relation has been used in the "ionization method" aiming to determine the mass of the black holes, and it gives results compatible with the reverberation mapping method (Wandel et al. 1999), but more uncertain.

Thus a possible interpretation of the observed correlation is that among clouds released by the gravitationally unstable disc, only those located at the right distance (i.e. corresponding to the right ionization parameter or ionizing flux) are observed as BLR clouds: the others could contribute to the Warm Absorber. This could explain why $R_{\text {crit }}$ constitutes a "lower envelope" to $R_{\mathrm{BLR}}$ for a given $\mathrm{BH}$ mass. Also since a constant ionizing flux corresponds to $R_{\mathrm{BLR}} / R_{\mathrm{G}} \propto \sqrt{\dot{m} / M}$, we see that the objects displaying the largest $R_{\mathrm{BLR}} / R_{\mathrm{G}}$ ratios, hence the smaller line widths, are those which have the largest accretion rates in Eddington unit and the smallest masses. Such a situation is proposed to explain the properties of NLS1s. Note that in this picture, the more intense soft X-ray excess displayed by NLS1s compared to BLS1s can also play a role, since such a spectrum has a stronger ionizing power for the same ionization parameter (e.g. Wandel 1997).

\section{Conclusion}

We have discussed the relations between the optical luminosity, the BH mass, and the size of the BLR, in the framework of the standard accretion disc model.

We have shown first that the optical luminosity cannot be accounted for, meaning that the standard accretion disc picture (stationary, geometrically thin and optically thick) does not hold, at least in the region emitting the optical band, i.e. at $\sim 100 R_{\mathrm{G}}$. Either the major fraction of the optical luminosity is not due to disc emission, or the disc is not "standard": it is unstable, or the accretion rate depends on the radius, owing to strong outflows or to advection dominated accretion. In these last cases, the implied mass rate would have to be strongly super-critical. We would thus favor the first explanation. We have also shown that NLS1s are extreme in this context: either they have a larger fraction of non disc emission in the optical range and are radiating close to their Eddington luminosity, or they have strongly super-Eddington luminosity, which seems implausible. So again in this case it seems that the best explanation would be that a large fraction of the optical emission (larger even than for BLS1s) is not produced by the disc.

In the second part, we have studied the relation between the disc and the BLR, and we have shown that there is a good agreement between the size of the BLR and the critical radius at which an $\alpha$-disc becomes gravitationally unstable. This suggests that the BLR is produced above the gravitationally unstable part of the disc. However the ionization parameter should also play a role in explaining the correlation observed between the size and the luminosity. If BLR clouds are seen only for a small range of ionization parameters or of ionizing flux, the size of the BLR expressed in $R_{\mathrm{G}}$ should increase with the accretion rate expressed in Eddington units and decrease with the $\mathrm{BH}$ mass, according to the observed correlation. This could explain the small widths of NLS1s.

Acknowledgements. We are grateful to Martin Ward for a careful reading of the manuscript leading to several improvements.

\section{References}

Abramowicz, M. A., Czerny, B., Lasota, J. P., \& Szuszkiewicz, E. 1988, ApJ, 332, 646

Antonucci, R. R. J., \& Miller, J. S. 1985, ApJ, 297, 621

Boller, T., Brandt, W. N., \& Fink, H. 1996, A\&A, 305, 53

Boller, Th., Brandt, W. N., Leighly, K. M., \& Ward, M. J. 2000, Proceedings of the workshop on observational and theoretical progress in the study of NLS1 galaxies, held in Bad Honnef, Germany, Dec. 8-11 1999, New Astron. Rev., 44,381 
Cannizzo, J. K. 1993, ApJ, 419, 318

Celotti, A., Fabian, A. C., \& Rees, M. J. 1992, MNRAS, 255, 419

Collin-Souffrin, S., Joly, M., Pequignot, D., \& Dumont, S. 1986, A\&A, 166, 27

Collin-Souffrin, S., Czerny, B., Dumont, A.-M., \& Zycki, P. 1996, A\&A, 314, 393

Collin, S., \& Huré, J. 1999, A\&A, 341, 385

Collin, S., \& Zahn, J.-P. 1999, A\&A, 344, 433

Collin, S. 2000, to appear in The starburst-AGN connection, Advanced Lectures held in Tonantzintla, Mexico (Ed. World Scientific)

Dumont, A. M., \& Collin-Souffrin, S. 1990, A\&A, 229, 302

Edwards, A. C. 1980, MNRAS, 190, 757

Goldreich, P., \& Lynden-Bell, D. 1965, MNRAS, 130, 97

Hubeny, I., Agol, E., Blaes, O., \& Krolik, J. H. 2000, ApJ, 533, 710

Huré, J.-M., Collin-Souffrin, S., Le Bourlot, J., \& Pineau des Forêts, G. 1994, A\&A, 290, 19

Huré, J. 1998, A\&A, 337, 625

Huré, J.-M. 2000, A\&A, 358, 378

Kaspi, S., Smith, P. S., Netzer, H., Maoz, D., Jannuzi, B. T., \& Giveon, U. 2000, ApJ, 533, 631

Koratkar, A., \& Blaes, O. 1999, PASP, 111, 1

Krolik, J. H., McKee, C. F., \& Tarter, C. B. 1981, ApJ, 249, 422

Krolik, J. H., \& Begelman, M. C. 1988, ApJ, 329, 702

Kumar, P. 1999, ApJ, 519, 589

Laor, A., Fiore, F., Elvis, M., Wilkes, B. J., \& McDowell, J. C. 1997, ApJ, 477, 93

Lynden-Bell, D., \& Pringle, J. E. 1974, MNRAS, 168, 603

Malkan, M. A., \& Sargent, W. L. W. 1982, ApJ, 254, 22

Murray, N., \& Chiang, J. 1995, ApJL, 454, 105
Murray, N., \& Chiang, J. 1997, ApJ, 474, 91

Narayan, R., \& Yi, I. 1995, ApJ, 452, 71

Nicastro, F. 2000, ApJL, 530, 60

Paczynski, B. 1978, AcA, 28, 91

Penston, M. V. 1988, MNRAS, 233, 601

Peterson, B. M. 1993, PASP, 105, 247

Peterson, B. M., Wandel, A. 1999, ApJL, 521, 95

Peterson, B. M., Wanders, I., Bertram, R., et al. 1998, ApJ, 501, 82

Peterson, B. M., \& Wandel, A. 2000, ApJL, 540, 13

Peterson, B. M., et al. 2000, ApJ, 542, 161

Peterson, B. M. 2000, to appear in The starburst-AGN connection, Advanced Lectures held in Tonantzintla, Mexico (Ed. World Scientific)

Pounds, K. A., Done, C., \& Osborne, J. P. 1995, MNRAS, 277, L5

Puchnarewicz, E. M., Mason, K. O., Siemiginowska, A., et al. 2000, ApJ, in press [astro-ph/0011389]

Sanders, D. B., Phinney, E. S., Neugebauer, G., Soifer, B. T., \& Matthews, K. 1989, ApJ, 347, 29

Scoville, N. Z., \& Norman, C. 1988, ApJ, 332, 163

Shakura, N. I., \& Sunyaev, R. A. 1973, A\&A, 24, 337

Shields, G. A. 1978, Nature, 272, 423

Shlosman, I., \& Begelman, M. C. 1989, ApJ, 341, 685

Siemiginowska, A., Czerny, B., \& Kostyunin, V. 1996, ApJ, 458, 491

Shore, S. N., \& White, R. L. 1982, ApJ, 256, 390

Toomre, A. 1964, ApJ, 139, 1217

Wandel, A. 1997, ApJL, 490, 131

Wandel, A., Peterson, B. M., \& Malkan, M. A. 1999, ApJ, 526, 579

Witt, H. J., Czerny, B., \& Zicky, P. T. 1997, MNRAS, 286, 848 